\title{
Periductal Induction of High Endothelial Venule-Like Vessels in Type 1 Autoimmune Pancreatitis
}

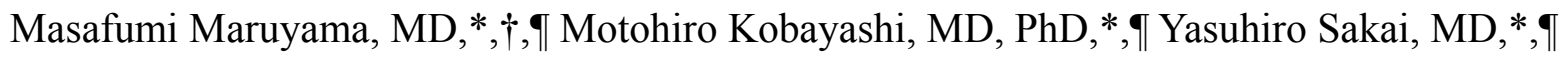

Nobuyoshi Hiraoka, MD, PhD, Ayumi Oya, MD, ${ }^{*}, \S$ Shunsuke Kageyama, MS,* Eiji Tanaka, MD, $\mathrm{PhD}, \dagger$ Jun Nakayama, MD, PhD,* and Toshio Morohoshi, MD, PhD\|

From the *Department of Molecular Pathology, Shinshu University Graduate School of Medicine, Matsumoto, Japan; $\uparrow$ Department of Medicine, Division of Hepatology and Gastroenterology, Shinshu University School of Medicine, Matsumoto, Japan; $₫$ Pathology Division, National Cancer Center Research Institute, Tokyo, Japan; §Department of Radiology, Shinshu University School of Medicine, Matsumoto, Japan; and ||Department of Pathology, Showa University School of Medicine, Tokyo, Japan.

TThese authors contributed equally to this work.

Correspondence: Motohiro Kobayashi, MD, PhD, Department of Molecular Pathology, Shinshu University Graduate School of Medicine, 3-1-1 Asahi, Matsumoto, Nagano 390-8621, Japan (e-mail: motokoba@shinshu-u.ac.jp). Telephone: +81-263-37-3395, Fax: +81-263-37-2581

Running title: High Endothelial Venule in Type 1 Autoimmune Pancreatitis

This work was supported in part by Grant-in-Aid for Young Scientists 22790343 from the Ministry of Education, Culture, Sports, Science and Technology of Japan (to M.K.).

Disclosure: The authors have no conflicts of interest to disclose. 


\section{Abstract}

Objectives: Type 1 autoimmune pancreatitis (AIP) is histologically characterized by dense lymphoplasmacytic infiltration and marked storiform fibrosis, manifestations associated with pancreatic ducts. Such periductal lymphocyte recruitment is thought to be elicited by dysregulation of mechanisms governing physiological lymphocyte homing. The present study was undertaken to determine whether vascular addressins including peripheral lymph node addressin (PNAd) and mucosal addressin cell adhesion molecule 1 (MAdCAM-1) play a role in type 1 AIP histogenesis.

Methods: Tissue sections of type 1 AIP and tumor-associated non-AIP chronic pancreatitis, as well as normal pancreas, were subjected to immunohistochemical analysis using vascular addressin-related antibodies.

Results: The number of periductal MECA- $79^{+}$high endothelial venule (HEV)-like vessels was increased in type 1 AIP relative to that seen in non-AIP chronic pancreatitis, while the number of MAdCAM- $1^{+} \mathrm{HEV}-$ like vessels did not differ between the two conditions. MECA-79 antigens are expressed on duct-forming epithelial cells not only in pancreas but also in salivary glands, which often harbor extrapancreatic lesions in type 1 AIP.

Conclusions: Type 1 AIP can be characterized by periductal induction of MECA- $79^{+} \mathrm{HEV}$-like vessels.

MECA-79 ${ }^{+}$6-sulfo sialyl Lewis X-related carbohydrate antigens expressed on duct-forming epithelial 
cells could be associated with type 1 AIP pathogenesis.

Keywords: type 1 autoimmune pancreatitis (AIP); IgG4-related disease; high endothelial venule (HEV); peripheral lymph node addressin (PNAd); mucosal addressin cell adhesion molecule 1

(MAdCAM-1) 


\section{Introduction}

Autoimmune pancreatitis (AIP) is a chronic, relapsing inflammatory disorder commonly affecting the head of the pancreas and often resulting in stenosis/obstruction of the main pancreatic duct with obstructive jaundice [1,2]. Yoshida et al characterized AIP in 1995 [3], but it was not until in 2001 that Hamano et al observed elevated levels of serum IgG4 in AIP [4]. Subsequently, the observation of increased numbers of tissue $\mathrm{IgG}^{+}$plasma cells has emerged as a histological marker of the condition [5]. However, although current evidence strongly suggests an autoimmune basis for AIP $[6,7]$, its etiopathogenesis is not fully understood.

It has been noticed that clinically diagnosed AIP is not a homogenous disease [8] but instead consists of at least two different groups: one "lymphoplasmacytic sclerosing pancreatitis (LPSP)", and the other "idiopathic duct-centric chronic pancreatitis (IDCP)" [9]. LPSP, also called as type 1 AIP, is characterized histologically by dense lymphoplasmacytic infiltration, marked storiform fibrosis, and obliterative phlebitis [10]. These findings are associated with large- and medium-sized interlobular ducts [2,9]. In addition to IgG4 $4^{+}$plasma cells, lymphoplasmacytic infiltration in type 1 AIP consists mainly of T cells [7], including T helper 2 (Th2) and regulatory T (Treg) [11] cells. Such lymphocyte recruitment is thought to be elicited by mechanisms similar to those operating in physiological lymphocyte homing, but in an unphysiological manner. 
Under physiological conditions, lymphocyte homing in secondary lymphoid organs is tightly regulated by a multistep process mediated by sequential adhesive interactions between circulating lymphocytes and specialized endothelial cells comprising high endothelial venules (HEVs) $[12,13]$. In the case of gut-associated lymphoid tissue (GALT), such as Peyer's patches and mesenteric lymph nodes, at least one of the following two interactions is required for initial "tethering and rolling": one via L-selectin and 6-sulfo sialyl Lewis X (sLeX)-capped glycoproteins recognized by the MECA-79 antibody (collectively called peripheral lymph node addressin (PNAd) [14-16]) and the other via $\alpha 4 \beta 7$ integrin and mucosal addressin cell adhesion molecule 1 (MAdCAM-1) [17,18]. These interactions trigger chemokine-dependent activation, integrin-mediated firm attachment to the endothelium, and transmigration of lymphocytes across blood vessels $[12,13]$.

Vascular addressins are expressed not only on HEVs in secondary lymphoid organs but also on HEV-like vessels induced in various non-lymphoid organs under chronic inflammatory states including rheumatoid arthritis, chronic Helicobacter pylori gastritis, and inflammatory bowel disease [19-23]. In this context, we previously reported that the number of MECA-79 $\mathrm{HEV-like} \mathrm{vessels}$ increases in active ulcerative colitis (UC) relative to that seen in the remission phase of UC, and that such an increase is associated with elevations of transcripts encoding $N$-acetylglucosamine-6-O-sulfotransferase 1 (GlcNAc6ST-1) [24-26]. Our subsequent study revealed 
that the number of MAdCAM- $1^{+} \mathrm{HEV}$-like vessels is increased in UC relative to normal colonic

mucosa, although the number of these vessels does not differ between active and remission phases of

UC, suggesting that UC disease activity is not enhanced by MAdCAM-1 protein expression but rather

by L-selectin ligand carbohydrate 6-sulfo sLeX displayed on that protein $[27,28]$.

Here we show that MECA-79 ${ }^{+} \mathrm{HEV}$-like vessels are preferentially induced around medium-

to large-sized interlobular ducts in type 1 AIP, an observation that could aid histological diagnosis of

type 1 AIP. Moreover, we found that the MECA-79 antigen is also expressed on duct-forming

epithelial cells not only in pancreas but also in salivary glands, which are often involved in type 1 AIP

as an extrapancreatic lesion. Considering the distribution of MECA-79 antigen on exocrine

duct-forming epithelium in organs susceptible to type 1 AIP, 6-sulfo sLeX-related carbohydrate

antigens displayed on these epithelial cells could be associated with type 1 AIP pathogenesis.

\section{Materials and methods}

\section{Human Tissue Samples}

Formalin-fixed paraffin-embedded tissue blocks of surgically resected pancreas from patients with type

1 AIP $(n=15)$ or with tumor-associated non-AIP chronic pancreatitis (CP) $(n=16)$, as well as normal

pancreas tissues $(n=3)$ were obtained from pathological archives at Shinshu University Hospital and 
the National Cancer Center Hospital. All type 1 AIP cases showed histological findings of LPSP with IgG4 ${ }^{+}$plasma cell infiltrate (Figure 1). Tissue block of salivary glands with IgG4-related sclerosing sialadenitis $(n=3)$ and normal salivary glands adjacent to Warthin's tumor $(n=10)$ were also subjected to immunohistochemistry. Tissue blocks of tumor-associated non-AIP CP were chosen from surgically resected pancreas with invasive ductal carcinoma. The distal side of carcinomatous lesions showed chronic obstructive pancreatitis due to the stenosis of the main pancreatic duct. No tumor-like lesions composed of abscess or granulation tissue were observed. The analysis of human tissue samples was approved by the Ethics Committee of Shinshu University School of Medicine and the National Cancer Center.

\section{Immunohistochemistry}

Primary antibodies used in this study are listed in Table 1. Conventional immunohistochemistry for CD34 and MAdCAM-1 was carried out using the EnVision system (Dako, Glostrup, Denmark), and that for IgG1 and IgG4 was done by an indirect method. Double immunohistochemistry for MECA-79 and cytokeratins was performed as described [29] with modification. Briefly, after antigens were retrieved by boiling sections in $10 \mathrm{mM}$ Tris/HCl buffer ( $\mathrm{pH}$ 8.0) containing $1 \mathrm{mM}$ EDTA for $25 \mathrm{~min}$ in a microwave oven, endogenous peroxidase activity was quenched by soaking sections in 
absolute methanol containing $0.3 \%$ hydrogen peroxide for $30 \mathrm{~min}$. After blocking with $1 \%$ bovine serum albumin (BSA) in Tris-buffered saline (TBS) (pH 7.6) for $15 \mathrm{~min}$, sections were incubated with MECA-79 antibody at $4^{\circ} \mathrm{C}$ overnight followed by incubation with horseradish peroxidase (HRP)-conjugated anti-rat immunoglobulins (1:200; Dako) for $60 \mathrm{~min}$. The color reaction was developed with 3,3'-diaminobenzidine (DAB). To dissociate bound antibodies, sections were boiled in $10 \mathrm{mM}$ Tris/ $\mathrm{HCl}(\mathrm{pH} 8.0)$ containing $1 \mathrm{mM}$ EDTA in a microwave oven for $15 \mathrm{~min}$. Sections were then incubated with AE1/AE3 for 60 min followed by incubation with HRP EnVision (Dako) for 30 min. The color reaction was developed with ImmPACT VIP peroxidase substrate (Vector Laboratories, Burlingame, CA). Sections were briefly counterstained with hematoxylin. For dual immunofluorescence staining, Alexa Fluor 488-conjugated anti-rat IgM and Alexa Fluor 555-conjugated anti-mouse IgG (both 1:1000; Invitrogen, Carlsbad, CA) were used as secondary antibodies, and sections were mounted with Vectashield mounting medium with DAPI (Vector Laboratories).

Because the appearance of HEV-like vessels in type 1 AIP was most prominent around medium- to large-sized interlobular ducts where dense lymphoplasmacytic infiltration was observed, we evaluated the number of HEV-like vessels in these areas, while such periductal association was not observed in non-AIP CP. Immunostained sections were observed under a light microscope at a 
magnification of $\times 200$. In each specimen, five fields of periductal areas with most prominent

appearance of $\mathrm{HEV}$-like vessels were chosen, and the number of respective CD34 ${ }^{+}, \mathrm{MAdCAM}-1^{+}$, and MECA- $79^{+}$vessels was counted. MAdCAM- $1^{+} / \mathrm{CD} 34^{+}$and $\mathrm{MECA}-79^{+} / \mathrm{CD} 34^{+}$vessel ratios were calculated as described previously [20,22].

$\underline{\text { Statistical Analysis }}$

Data are expressed as means \pm SD. Differences among groups were statistically analyzed by one-way analysis of variance (ANOVA) with a Bonferroni post-test using InStat3 software (GraphPad Software, San Diego, CA). $\quad$ p values less than 0.05 were considered significant.

\section{Results}

Expression of MECA-79 Antigen on Epithelial Cells Forming Pancreatic Ducts

Initially, we carried out immunohistochemistry for MECA-79 to detect HEV-like vessels in type 1 AIP. However, in an unanticipated finding, we observed MECA-79 stained not only HEV-like vessels but also in a significant proportion of pancreatic ducts (Figure 2, left panels). This finding hampered identification of HEV-like vessels, as it was difficult to distinguish HEV-like vessels from small-sized interlobular and intercalated ducts, particularly those composed of flattened epithelial cells. To 
overcome this difficulty, we performed dual immunofluorescence staining with MECA-79 and with AE1/AE3, which reacts with cytokeratins expressed in epithelial cells, including those comprising pancreatic ducts (Figure 2, upper middle panel), but not with endothelial cells (Figure 2, lower middle panel). This staining enabled us to distinguish MECA-79 ${ }^{+}$HEV-like vessels from MECA-79 ${ }^{+}$ pancreatic ducts (Figure 2, right panels).

\section{Prominence of Periductal MECA-79 $9^{+}$HEV-Like Vessels in Type 1 AIP}

We next performed double immunohistochemistry employing non-fluorescent chromogenic substrates to analyze pancreatic tissues with type 1 AIP or non-AIP chronic pancreatitis (CP), as well as normal pancreas. In type $1 \mathrm{AIP}$ (Figure $3 \mathrm{a}$ and $\mathrm{b}$, left panels), prominent lymphoplasmacytic infiltration with germinal center formation was observed diffusely around medium- to large-sized interlobular ducts, where significant numbers of MECA-79 ${ }^{+} \mathrm{HEV}$-like vessels were detected. Besides these vessels, MAdCAM- $1^{+} \mathrm{HEV-like} \mathrm{vessels} \mathrm{were} \mathrm{also} \mathrm{frequently} \mathrm{observed.} \mathrm{Basically,} \mathrm{there} \mathrm{was} \mathrm{no} \mathrm{significant}$ difference in the distribution between MECA- $79^{+}$and MAdCAM- $1^{+}$vessels. In addition to periductal areas, these HEV-like vessels were also found in lymphoplasmacytic aggregates formed in peripancreatic adipose tissues (data not shown). By contrast, in non-AIP CP (Figure 3a and b, middle panels), small patchy lymphocyte aggregates were observed, but MECA-79 ${ }^{+} \mathrm{HEV}-$ like vessels were 
scarcely detected, while MAdCAM- $1^{+} \mathrm{HEV}$-like vessels were frequently observed. In normal

pancreas, both MECA $-79^{+}$and MAdCAM- ${ }^{+} \mathrm{HEV}$-like vessels were barely detected (Figure $3 \mathrm{a}$ and $\mathrm{b}$, right panels).

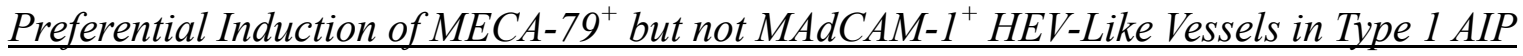

We next evaluated percentages of respective MECA- $79^{+}$and MAdCAM- ${ }^{+}$vessels among CD34 ${ }^{+}$

vessels in type 1 AIP, non-AIP CP, and normal pancreatic tissue. Percentages of MAdCAM- $1^{+}$vessels in both type 1 AIP and non-AIP CP were significantly higher than those seen in normal pancreas, although these percentages did not differ significantly between the two pathological conditions (Figure 4, left). On the other hand, the percentage of MECA $-79^{+}$vessels in type 1 AIP was not only higher than that seen in normal pancreas but also higher than that seen in non-AIP CP, with high statistical significance (Figure 4, right). Percentages of MECA- $79^{+}$vessels between non-AIP CP and normal pancreas did not differ significantly. Taken together, these findings indicate that MAdCAM-1 proteins are expressed on HEV-like vessels in chronic inflammatory states regardless of disease etiology, and that, in the case of type 1 AIP, MECA-79 antigens are further expressed on these vessels. 
The occasional observation of extrapancreatic lesions with type 1 AIP suggests that MECA-79 ${ }^{+}$

HEV-like vessels function in their histogenesis. As shown in Figure 5, sclerosing sialadenitis, an extrapancreatic type 1 AIP lesion involving salivary glands, showed histological similarity to LPSP, that is, dense lymphoplasmacytic infiltration predominantly composed of $\mathrm{IgG}^{+}$plasma cells and storiform fibrosis resulting in acinar cell atrophy. Interestingly, similar to LPSP, medium- to large-sized interlobular ducts were also MECA-79-positive, and significant numbers of MECA-79 HEV-like vessels were observed around these ducts. Moreover, expression of MECA-79 antigens on duct-forming epithelial cells was observed not only in sclerosing sialadenitis but also in normal salivary glands (data not shown). These findings suggest that dense lymphoplasmacytic infiltration commonly seen in LPSP and sclerosing sialadenitis may be promoted by MECA-79 ${ }^{+}$HEV-like vessels induced around exocrine ducts.

\section{Discussion}

The present study demonstrates that the occurrence of periductal MECA-79 ${ }^{+} \mathrm{HEV}$-like vessels is a prominent feature of type 1 AIP. As noted, type 1 AIP is histologically characterized by dense periductal lymphoplasmacytic infiltration, storiform fibrosis, and obliterative phlebitis - hence the name LPSP (for lymphoplasmacytic sclerosing pancreatitis) [10]. Here, we hypothesized that dense 
lymphoplasmacytic infiltration is brought on via HEV-like vessels, a condition observed in other non-lymphoid organs under chronic inflammatory conditions [19-21]. As predicted, prominent MECA- $79^{+}$HEV-like vessels were observed in type 1 AIP mainly around interlobular ducts where dense lymphoplasmacytic infiltration occurs. Such periductal induction of MECA-79 ${ }^{+}$HEV-like vessels was also observed in sclerosing sialadenitis, an extrapancreatic lesion of type 1 AIP. This finding is consistent with the current consensus that type 1 AIP is a manifestation of IgG4-related disease [30].

IgG4-related disease could be immunopathologically characterized by the over-production of Th2 and regulatory cytokines such as interleukin (IL)-10 and transforming growth factor (TGF)- $\beta$ secreted by Treg cells [11]. IL-10 may participate in the elevation of serum IgG4 concentration and IgG4-positive plasma cell infiltration, because IL-10 has a major role in directing B cells to produce IgG4 [31]. In this context, such IL-10-secreting Treg cells in affected tissues could be recruited via MECA- $79^{+}$HEV-like vessels.

In the present study, we found that the number of periductal MECA- $79^{+} \mathrm{HEV}-$ like vessels in type 1 AIP was significantly greater than that seen in non-AIP CP, while the number of MAdCAM- ${ }^{+}$ vessels did not differ between the two diseases. This finding confirms our previous study on UC demonstrating that the number of MECA-79 ${ }^{+}$HEV-like vessels in active phase UC exceeds that seen in 
remission phase UC, while the number of MAdCAM- $1^{+} \mathrm{HEV}$-like vessels did not differ between the two phases [28]. Overall, these findings suggest that MAdCAM-1 proteins are expressed on HEV-like vessels in chronic inflammatory states regardless of disease etiology, and that, in the case of type 1 AIP, MAdCAM-1 proteins and/or other scaffold proteins such as CD34 on these HEV-like vessels are preferentially post-translationally glycosylated with 6-sulfo sLeX-related carbohydrates $[27,28]$. However, it is possible that the significant increase of MECA-79 ${ }^{+} \mathrm{HEV}-$ like vessels in type 1 AIP, compared to non-AIP CP, might be brought on by the intense inflammation seen in type 1 AIP. Indeed, in type 1 AIP, we observed more number of MECA-79 ${ }^{+} \mathrm{HEV}$-like vessels in areas with intense lymphoplasmacytic infiltration compared to that in areas with less intense inflammation (data not shown). Further studies are necessary to clarify whether the increase of MECA-79 ${ }^{+}$HEV-like vessels is simply associated with the magnitude of inflammation.

It is of interest whether steroid treatment alters expression levels of MECA-79 antigens on HEV-like vessels since type 1 AIP and its extrapancreatic lesions generally show favorable response to steroid therapy [30]. In our previous study on chronic H. pylori gastritis [22], we have shown that MECA-79 antigens on HEV-like vessels are disappeared after eradication of $H$. pylori using antibiotics.

This fact indicates that expression of MECA-79 antigens on HEV-like vessels can be abolished by removing causes that led to their expression. It is tempting to speculate that the steroid 
responsiveness of AIP might be brought on, in part, by the suppression of glycosyltransferases and/or sulfotransferases involved in the biosynthesis of MECA-79 antigens and, thus, reduced expression levels of MECA-79 antigens on HEV-like vessels.

In contrast to LPSP (type 1 AIP), neutrophilic infiltration within the epithelium and ductal lumen, a condition known as "granulocyte epithelial lesion (GEL)", in the main and/or interlobular ducts is a characteristic of the other subtype of AIP, IDCP (type 2 AIP) [32]. Since neutrophilic infiltration at the inflammation site is elicited chiefly by interaction between non-sulfated sLeX on neutrophils and E-selectin on endothelial cells $[33,34]$, mechanisms other than the L-selectin/PNAd system may operate in IDCP histogenesis.

In our sclerosing sialadenitis cases examined, ductal (not periductal) inflammation was minimal, while the other histological findings were similar to those seen in type 1 AIP. According to the report by Laco et al [35], however, lymphocyte infiltration to the duct epithelium was seen in three out of six cases. Moreover, by combining their six cases with previously reported 28 cases of IgG4-related sclerosing sialadenitis [36,37], they concluded that well-formed lymphoepithelial island were seen in only two out of 34 cases. These facts suggest that ductal inflammation is not an essential finding in making a histopathological diagnosis of IgG4-related sclerosing sialadenitis.

In the present study, we also found that MECA-79 antigen is expressed not only on HEV-like 
vessels but also on the epithelium forming pancreatic and salivary ducts. The function of 6-sulfo sLeX-related carbohydrates in these epithelial cells is currently unknown. Further studies are required to clarify whether 6-sulfo sLeX-related carbohydrates play a role, possibly as autoantigens, in the pathogenesis of type 1 AIP.

\section{Acknowledgements}

We thank Dr. Minoru Fukuda for encouragement, Ms. Yasuyo Shimojo for technical assistance, and Dr.

Elise Lamar for critical reading of the manuscript. 


\section{References}

1. Klöppel G, Lüttges J, Löhr M, et al. Autoimmune pancreatitis: pathological, clinical, and immunological features. Pancreas. 2003;27:14-19.

2. Klöppel G. Chronic pancreatitis, pseudotumors and other tumor-like lesions. Mod Pathol. 2007;20

Suppl 1:113-131.

3. Yoshida K, Toki F, Takeuchi T, et al. Chronic pancreatitis caused by an autoimmune abnormality.

Proposal of the concept of autoimmune pancreatitis. Dig Dis Sci. 1995;40:1561-1568.

4. Hamano H, Kawa S, Horiuchi A, et al. High serum IgG4 concentrations in patients with sclerosing pancreatitis. N Engl J Med. 2001;344:732-738.

5. Deshpande V, Chicano S, Finkelberg D, et al. Autoimmune pancreatitis: a systemic immune complex mediated disease. Am J Surg Pathol. 2006;30:1537-1545.

6. Kino-Ohsaki J, Nishimori I, Morita M, et al. Serum antibodies to carbonic anhydrase I and II in patients with idiopathic chronic pancreatitis and Sjögren's syndrome. Gastroenterology.

1996;110:1579-1586.

7. Okazaki K, Uchida K, Ohana M, et al. Autoimmune-related pancreatitis is associated with autoantibodies and a Th1/Th2-type cellular immune response. Gastroenterology. 2000;118:573-581.

8. Deshpande V, Gupta R, Sainani N, et al. Subclassification of autoimmune pancreatitis: a histologic 
classification with clinical significance. Am J Surg Pathol. 2011;35:26-35.

9. Notohara K, Burgart LJ, Yadav D, et al. Idiopathic chronic pancreatitis with periductal lymphoplasmacytic infiltration: clinicopathologic features of 35 cases. Am J Surg Pathol.

2003;27:1119-1127.

10. Kawaguchi K, Koike M, Tsuruta K, et al. Lymphoplasmacytic sclerosing pancreatitis with cholangitis: a variant of primary sclerosing cholangitis extensively involving pancreas. Hum Pathol. 1991;22:387-395.

11. Zen Y, Fujii T, Harada K, et al. Th2 and regulatory immune reactions are increased in immunoglobulin G4-related sclerosing pancreatitis and cholangitis. Hepatology. 2007;45:1538-1546.

12. Springer TA. Traffic signals for lymphocyte recirculation and leukocyte emigration: the multistep paradigm. Cell. 1994;76:301-314.

13. Butcher EC, Picker LJ. Lymphocyte homing and homeostasis. Science. 1996;272:60-66.

14. Streeter PR, Rouse BT, Butcher EC. Immunohistologic and functional characterization of a vascular addressin involved in lymphocyte homing into peripheral lymph nodes. J Cell Biol.

1988;107:1853-1862.

15. Yeh JC, Hiraoka N, Petryniak B, et al. Novel sulfated lymphocyte homing receptors and their control by a core 1 extension $\beta 1,3-N$-acetylglucosaminyltransferase. Cell. 2001;105:957-969. 
16. Rosen SD. Ligand for L-selectin: homing, inflammation, and beyond. Annu Rev Immunol.

2004;22:129-156.

17. Berlin C, Berg EL, Briskin MJ, et al. $\alpha 4 \beta 7$ integrin mediates lymphocyte binding to the mucosal

vascular addressin MAdCAM-1. Cell. 1993;74:185-195.

18. Hoshino $\mathrm{H}$, Kobayashi M, Mitoma J, et al. An integrin $\alpha 4 \beta 7 \cdot \mathrm{IgG}$ heterodimeric chimera binds to

MAdCAM-1 on high endothelial venule in gut-associated lymphoid tissue. J Histochem Cytochem.

2011;59:572-583.

19. Michie SA, Streeter PR, Bolt PA, et al. The human peripheral lymph node vascular addressin. An inducible endothelial antigen involved in lymphocyte homing. Am J Pathol. 1993;143:1688-1698.

20. Renkonen J, Tynninen O, Häyry P, et al. Glycosylation might provide endothelial zip codes for organ-specific leukocyte traffic into inflammatory sites. Am J Pathol. 2002;161:543-550.

21. Aloisi F, Pujol-Borrell R. Lymphoid neogenesis in chronic inflammatory diseases. Nat Rev

Immunol. 2006;6:205-217.

22. Kobayashi M, Mitoma J, Nakamura N, et al. Induction of peripheral lymph node addressin in human gastric mucosa infected by Helicobacter pylori. Proc Natl Acad Sci U S A.

2004;101:17807-17812.

23. Kobayashi M, Mitoma J, Hoshino H, et al. Prominent expression of sialyl Lewis X-capped core 
2-branched $O$-glycans on high endothelial venule-like vessels in gastric MALT lymphoma. J Pathol.

2011;224:67-77.

24. Uchimura K, Muramatsu H, Kaname T, et al. Human $N$-acetylglucosamine-6- $O$-sulfotransferase involved in the biosynthesis of 6-sulfo sialyl Lewis X: molecular cloning, chromosomal mapping, and expression in various organs and tumor cells. J Biochem. 1998;124:670-678.

25. Suzawa K, Kobayashi M, Sakai Y, et al. Preferential induction of peripheral lymph node addressin on high endothelial venule-like vessels in the active phase of ulcerative colitis. Am J Gastroenterol.

2007;102:1499-1509.

26. Fujiwara M, Kobayashi M, Hoshino H, et al. Expression of long-form

$\mathrm{N}$-acetylglucosamine-6-O-sulfotransferase 1 in human high endothelial venules. J Histochem

Cytochem. in press.

27. Berg EL, McEvoy LM, Berlin C, et al. L-selectin-mediated lymphocyte rolling on MAdCAM-1.

Nature. 1993;366:695-698.

28. Kobayashi M, Hoshino H, Masumoto J, et al. GlcNAc6ST-1-mediated decoration of MAdCAM-1

protein with L-selectin ligand carbohydrates directs disease activity of ulcerative colitis. Inflamm

Bowel Dis. 2009;15:697-706.

29. Kobayashi M, Nakayama J. Immunohistochemical analysis of carbohydrate antigens in chronic 
inflammatory gastrointestinal diseases. Methods Enzymol. 2010;479:271-289.

30. Okazaki K, Uchida K, Koyabu M, et al. Recent advances in the concept and diagnosis of autoimmune pancreatitis and IgG4-related disease. J Gastroenterol. 2011;46:277-288.

31. Jeannin P, Lecoanet S, Delneste $\mathrm{Y}$, et al. IgE versus IgG4 production can be differentially regulated by IL-10. J Immunol. 1998;160:3555-3561.

32. Zamboni G, Lüttges J, Capelli P, et al. Histopathological features of diagnostic and clinical relevance in autoimmune pancreatitis: a study on 53 resection specimens and 9 biopsy specimens.

Virchows Arch. 2004;445:552-563.

33. Fukuda M, Spooncer E, Oates JE, et al. Structure of sialylated fucosyl lactosaminoglycan isolated from human granulocytes. J Biol Chem. 1984;259:10925-10935.

34. Bevilacqua MP, Pober JS, Mendrick DL, et al. Identification of an inducible endothelial-leukocyte adhesion molecule. Proc Natl Acad Sci U S A. 1987;84:9238-9242.

35. Laco J, Ryska A, Celakovsky P, et al. Chronic sclerosing sialadenitis as one of the immunoglobulin G4-related diseases: a clinicopathological study of six cases from central Europe. Histopathology.

$2011 ; 58: 1157-1163$.

36. Kitagawa S, Zen Y, Harada K, et al. Abundant IgG4-positive plasma cell infiltration characterizes chronic sclerosing sialadenitis (Küttner’s tumor). Am J Surg Pathol. 2005;29:783-791. 
37. Geyer JT, Ferry JA, Harris NL, et al. Chronic sclerosing sialadenitis (Küttner tumor) is an IgG4-associated disease. Am J Surg Pathol. 2010;34:202-210. 


\section{Figure Legends}

Figure 1. Type 1 AIP showing histological findings of LPSP. (a) Hematoxylin and eosin (HE) staining of type 1 AIP showing lymphoplasmacytic infiltration with storiform fibrosis. (b,c)

Immunohistochemistry for IgG1 (b) and IgG4 (c), showing prominent infiltration of IgG4 ${ }^{+}$plasma cells.

Sections were counterstained with hematoxylin. $\quad$ Bar $=50 \mu \mathrm{m}$.

Figure 2. Dual immunofluorescence staining of type 1 AIP for MECA-79 (green) and AE1/AE3 (red).

Pancreatic ducts (PDs) are not only MECA-79-positive but also positive for AE1/AE3, which reacts

with cytokeratins expressed in epithelial cells. By contrast, HEVs and HEV-like vessels are positive

for MECA-79 but not for AE1/AE3. Bar $=50 \mu \mathrm{m}$.

Figure 3. Representative histology of pancreas with type 1 AIP (left panels) and non-AIP CP (middle

panels), and of normal pancreas (right panels). (a) Serial tissue sections were stained with HE (upper

group of panels) and doubly immunostained with MECA-79 (brown) and AE1/AE3 (purple) (lower

group of panels). Note that MECA $-79^{+}$pancreatic ducts are masked by purple color, which makes

MECA-79 ${ }^{+}$HEV-like vessels easily-identifiable as brown structures (see also Figure 3b, bottom row).

Bar $=500 \mu \mathrm{m} . \quad$ (b) Enlarged images of boxes shown in (a). Tissue sections were further 
immunostained for CD34 as a marker of vascular endothelial cells (second row) and for MAdCAM-1

(third row). Note that periductal lymphoplasmacytic infiltration observed in type 1 AIP is associated with prominent periductal appearance of MECA-79 $\mathrm{HEV}$-like vessels (bottom row, left panel). Bar $=$ $100 \mu \mathrm{m}$.

Figure 4. Percentages of MAdCAM- $1^{+}$(left) and MECA- $79^{+}$(right) vessels induced around pancreatic ducts in normal pancreas (white), non-AIP CP (gray) and type 1 AIP (black). Data are presented as means $\pm \mathrm{SD}$ (normal, $n=3$; non-AIP CP, $n=16$; type 1 AIP, $n=15$ ). $\quad * * *, p<0.001$; **, $p<0.01 ;$ NS, not significant vs. type 1 AIP.

Figure 5. (a-c) Periductal induction of HEV-like vessels in sclerosing sialadenitis. Serial tissue sections were stained with HE (a), immunostained with MECA-79 (b), and doubly immunostained with MECA-79 (brown) and AE1/AE3 (purple) (c). Bar $=200 \mu \mathrm{m} . \quad$ (d-f) Prominent infiltration of $\mathrm{IgG}^{+}$plasma cells in sclerosing sialadenitis. Serial tissue sections were stained with $\mathrm{HE}(\mathbf{d})$ and immunostained for $\operatorname{IgG1}(\mathbf{e})$ and $\operatorname{IgG} 4(\mathbf{f}) . \quad B a r=50 \mu \mathrm{m}$. 

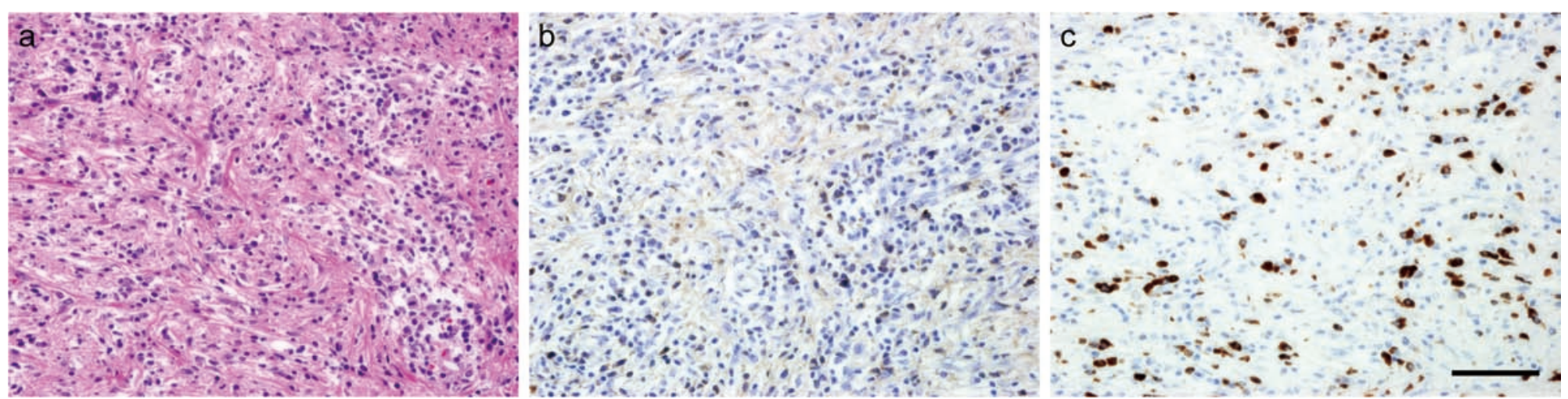


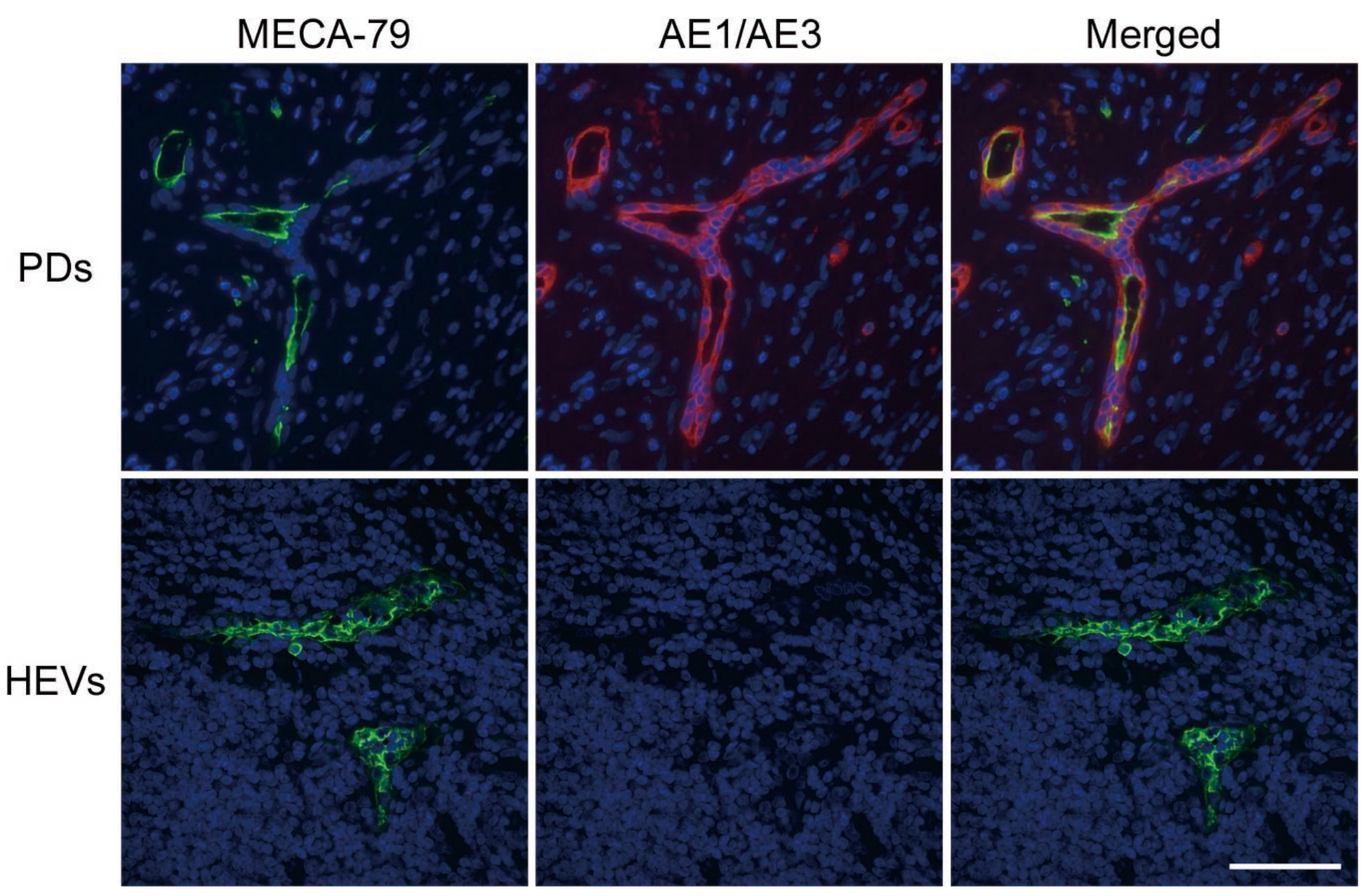


(a)

HE

MECA-79
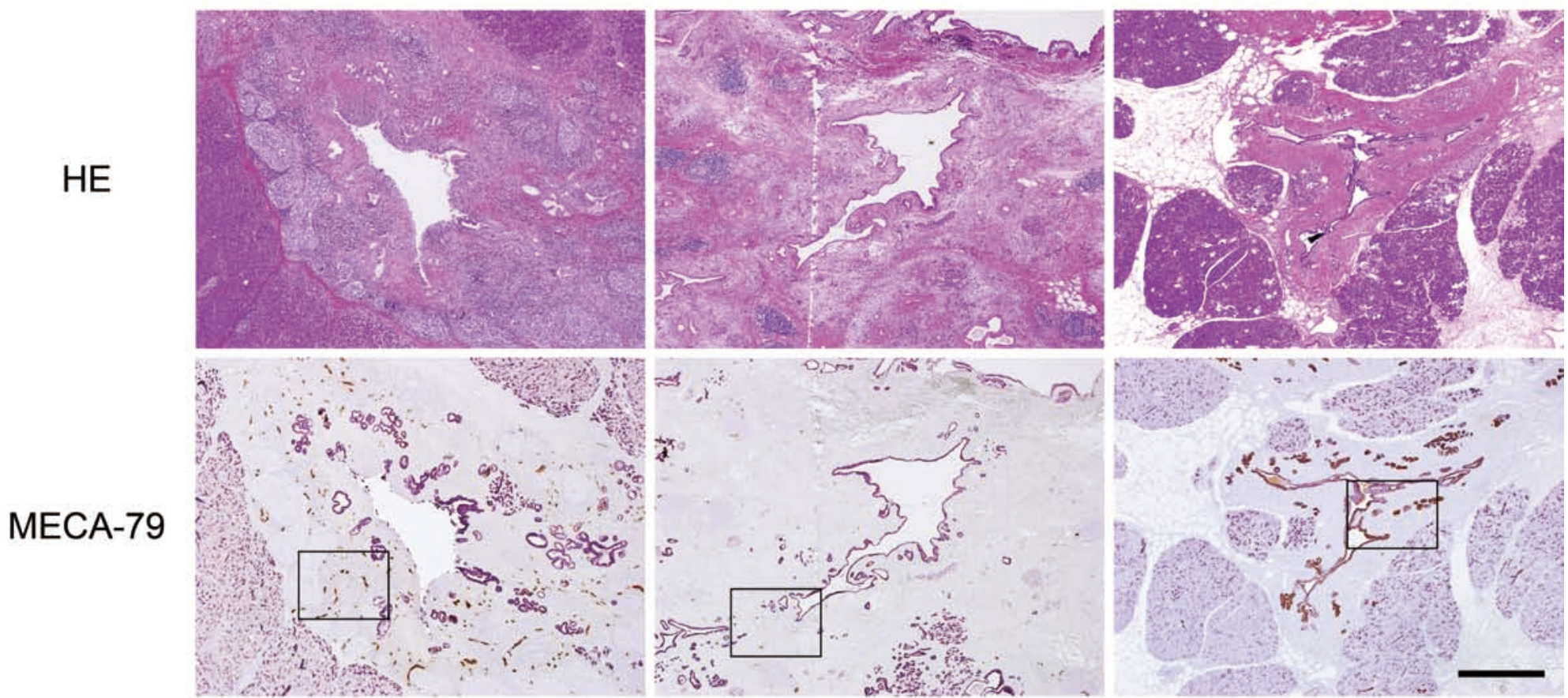

(b)

\section{AIP}

\section{Non-AIP CP}

\section{Normal}

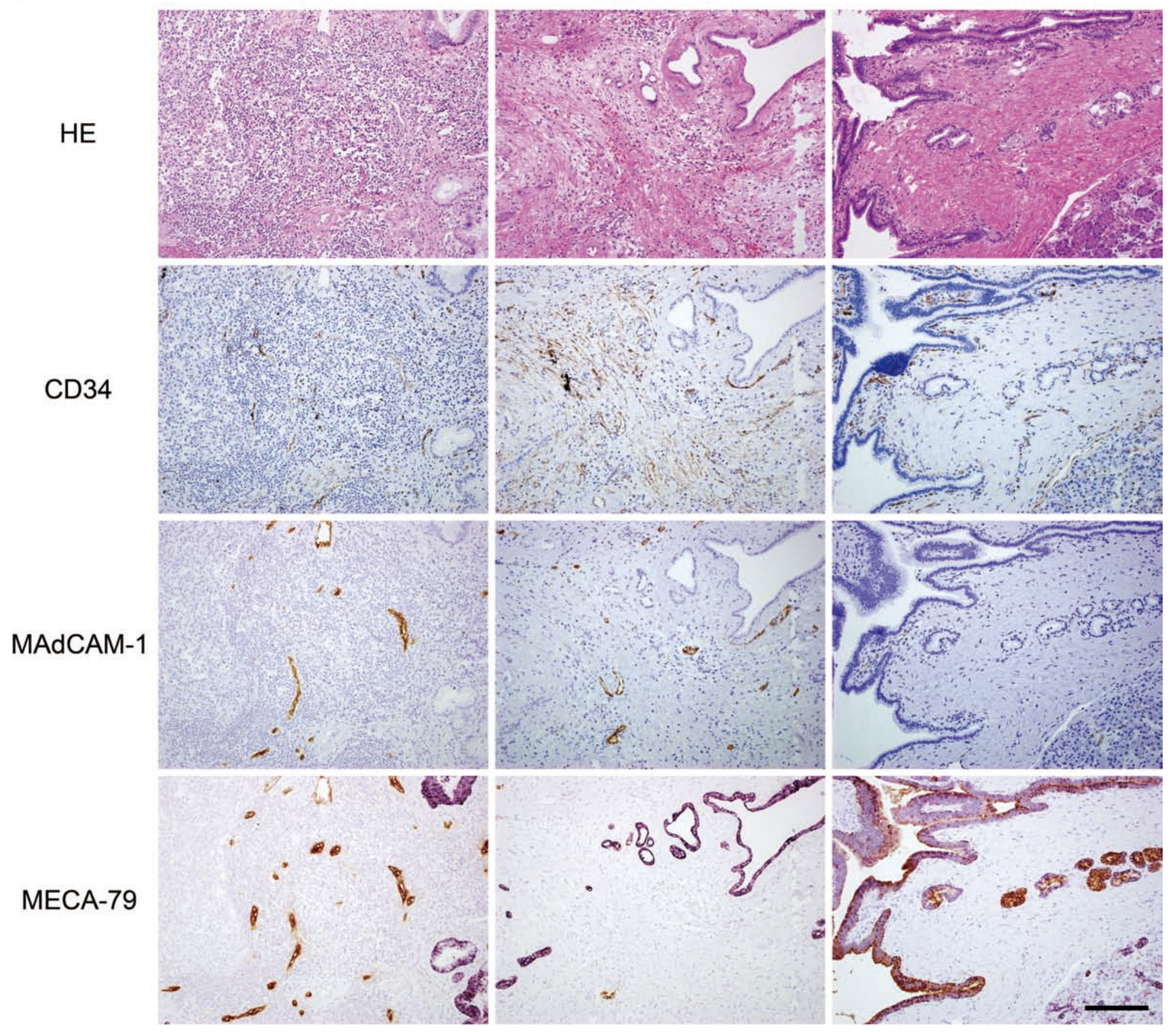




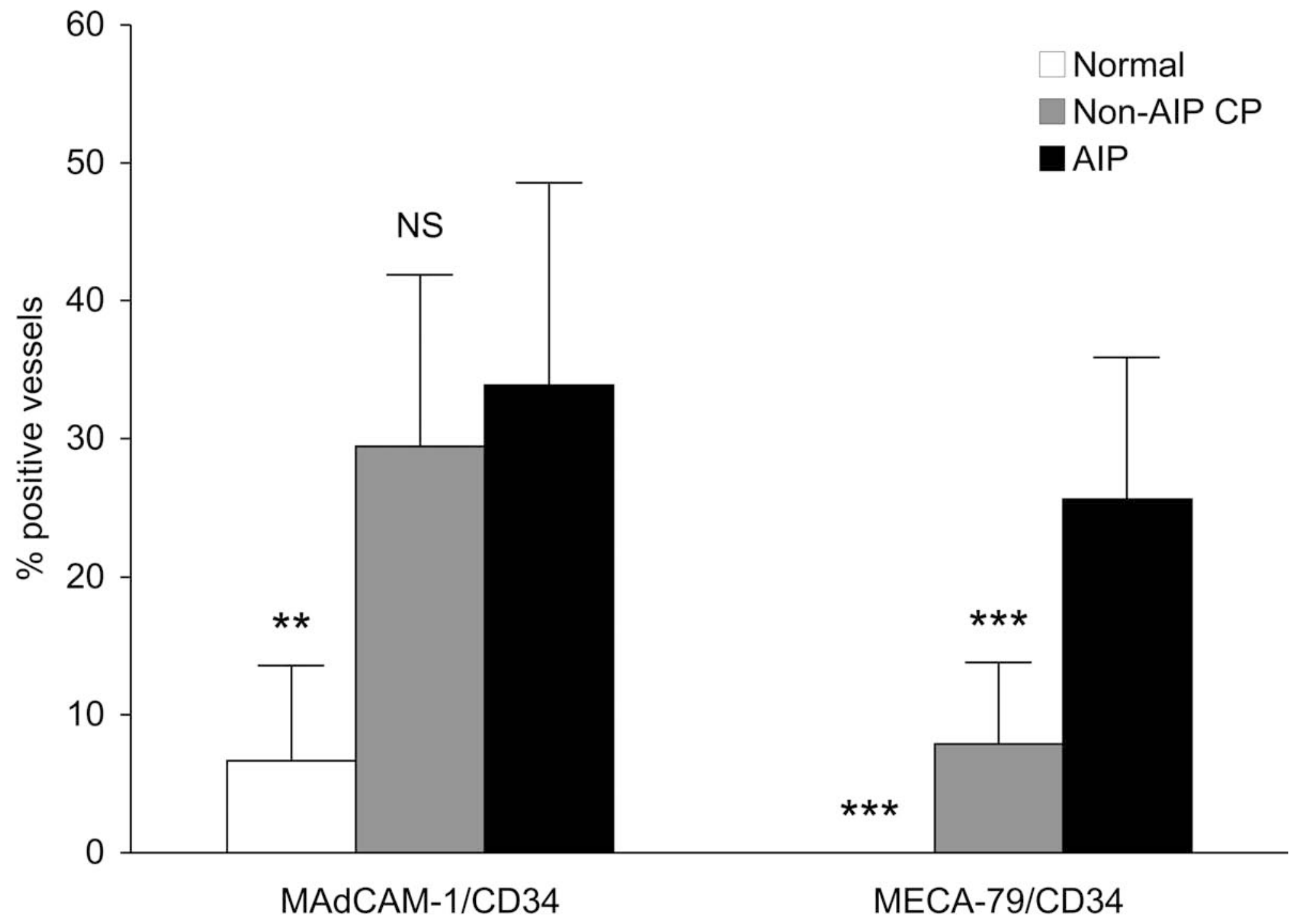




Table 1. Primary antibodies used in this study

\begin{tabular}{lllll}
\hline Name & Clone & Isotype & Source & Dilution \\
\hline Anti-human CD34 & QBEND10 & Mouse IgG & Immunotech, Marseille, France & $1: 200$ \\
Anti-human MAdCAM-1 & 1C3 & Mouse IgG & Arihiro et al. Pathol Int 2002; 52: 376-374 & $1: 400$ \\
Anti-PNAd & MECA-79 & Rat IgM & BD Pharmingen, San Diego, CA, USA & $1: 100$ \\
Anti-human cytokeratins & AE1/AE3 & Mouse IgG & Dako, Glostrup, Denmark & $1: 200$ \\
Anti-human IgG1 & - & Sheep polyclonal & Binding Site, Birmingham, UK & $1: 50$ \\
Anti-human IgG4 & - & Sheep polyclonal & Binding Site, Birmingham, UK & $1: 50$ \\
\hline
\end{tabular}

